\title{
Dielectric Study at Microwave Frequencies of Phase Transitions in Multiferroic Ceramics
}

\author{
B. Fraygola ${ }^{\text {a*, Adelino A. Coelho }}{ }^{\mathrm{b}}$, J. A. Eiras ${ }^{\mathrm{a}}$ \\ ${ }^{a}$ Grupo de Cerâmicas Ferroelétricas - GCFerr, Departamento de Física, \\ Universidade Federal de São Carlos - UFSCar, CEP 13565-670, São Carlos, SP, Brasil \\ ${ }^{\mathrm{b}}$ Laboratório de Preparação e Caracterização de Materiais - LPCM, \\ Departamento de Física - DFA, Instituto de Física "Gleb Wataghin” IFGW, \\ Universidade de Campinas - UNICAMP, Campinas, SP, Brasil
}

Received: December 17, 2012; Revised: April 15, 2013

\begin{abstract}
Multiferroic ceramic solid solutions between the ferroelectric relaxor $\mathrm{Pb}\left(\mathrm{Fe}_{2 / 3} \mathrm{~W}_{1 / 3}\right) \mathrm{O}_{3}(\mathrm{PFW})$ and "normal" ferroelectric $\mathrm{PbTiO}_{3}(\mathrm{PT})$ - PFW-PT have been synthesized by a modified B-site precursor method and characterized by microwave dielectric measurements. A dielectric dispersion occurring in the frequency range of $100 \mathrm{MHz}-3 \mathrm{GHz}$ and in a broad range of temperatures showed to be a powerful tool to detect electrostrictive and magnetostrictive effects. Anomalies in the temperature dependence of the dielectric parameters ( $f_{R}$ (characteristic frequency) and the dielectric strength $\Delta \varepsilon$ (characteristic of the dispersion)) have been observed at $\mathrm{T}_{\mathrm{N}}$ and $\mathrm{T}_{\mathrm{C}}$ for all samples, indicating that the coupling between the ferroelectric and antiferromagnetic orders occurs "via strain" and confirming the high sensitivity of microwave dielectric measurements to probe weak transitions in bulk ceramics.
\end{abstract}

Keywords: multiferroic, lead iron tungstate, microwave dielectric spectroscopy, phase transitions

\section{Introduction}

Ferroelectric materials present a dielectric dispersion in the frequency range of 0.1-1 GHz, which has been attributed to either a relaxation or a resonance process by different authors ${ }^{1-5}$. Nevertheless, the results reported in the literature have not been conclusive enough to distinguish clearly each process.

In spite of still not being clarified, several theoretical attempts have been proposed to explain the origin of this effect. The relation between the high frequency dielectric dispersion and the microstructure of ceramics was expressed by Von Hippe ${ }^{6}$ who attributed the piezoelectric resonance of the grain as the principal cause of the observed microwave anomalies. Kittel attributed the decrease of the dielectric constant at the microwave region to the resonance of the domain walls in ceramics ${ }^{7}$. Some others models concerning piezoelectric resonance of individual domains or on correlated hopping of off-centered ferroelectric active ions between several potential walls have been proposed. More recent experimental results confirms the elastic contributions to the high frequency dielectric anomalies, showing that ferroelectric and ferroelastic effects are always coupled each other and either mechanisms, resonance or relaxation-like, are always present without the presence of an intermediate case. For last, magnetoelectric coupling was only observed in the microwave dielectric measurements in many multiferroic materials, which simultaneously display electric dipole moment and magnetic ordering in the same phase ${ }^{8,9}$. At higher frequencies, conductive contributions to the dielectric permittivity can be minimized, suggesting microwave dielectric spectroscopy as an accurate tool to investigate phase transitions in multiferroic materials. On other hand,

\footnotetext{
*e-mail: barbarafraygola@gmail.com
}

the presence of an elastic subsystem in the crystal leads to the emergence of electrostriction which accompanies the FE ordering and changes the crystal size. Thus, in turn, changes the magnetic state due to magnetostriction, and vice versa. A systematic study of the elastic behavior also provides useful information for probing structural phase transitions as well as spin-phonon and electron-phonon coupling. Therefore, microwave dielectric spectroscopy reveals to be a powerful and accurate approach to investigate the ferroelastic contributions in multiferroic - magnetoelectric materials.

Among the single phase multiferroic materials, $\mathrm{Pb}\left(\mathrm{Fe}_{2 / 3} \mathrm{~W}_{1 / 3}\right) \mathrm{O}_{3}$-PFW shows both ferroelectric $\left(\mathrm{T}_{\mathrm{C}} \sim 180 \mathrm{~K}\right)$ and antiferromagnetic $\left(\mathrm{T}_{\mathrm{N}} \sim 340 \mathrm{~K}\right)$ properties ${ }^{10}$. These types of materials, called multiferroics have been used widely as multistate memory elements, electrically controlled ferromagnetic resonant systems, between others. PFW present a complex perovskite-type structure, in which the cations $\left(\mathrm{Fe}^{3+}, \mathrm{W}^{6+)}\right.$ are randomly distribute at the octahedral B-site positions. Besides, the Curie temperature is too low for practical applications. PFW can form solid solutions with $\mathrm{PbTiO}_{3}(\mathrm{PT})$ presenting changes of symmetry and a range of temperature, where magnetic and ferroelectric ordering coexists, depending on the PT contents. As the $\mathrm{PbTiO}_{3}$ PT content is increased, the Curie temperature increases, because PT has a transition at a rather high temperature (around $773 \mathrm{~K}$ ).

In current work, we report the magnetic and microwave dielectric characteristics of $\mathrm{Pb}\left(\mathrm{Fe}_{2 / 3} \mathrm{~W}_{1 / 3}\right) \mathrm{O}_{3} \mathrm{PbTiO}_{3}$ solid solutions multiferroics ceramics determined in the temperature range spanning both $\mathrm{T}_{\mathrm{N}}$ and $\mathrm{T}_{\mathrm{C}}$ in the frequency range of $1 \mathrm{MHz}-3 \mathrm{GHz}$. The microwave dielectric results characterized the magnetoelectric effect in the PFW-PT 
and demonstrated that the technique is powerful to detect magnetic transitions. Moreover, it was also verified that the magnetoelectric coupling can be arises indirectly via elastic contribution rather than a direct coupling between electrical and magnetic order parameters.

\section{Experimental Procedure}

$\mathrm{Fe}_{2} \mathrm{O}_{3}$ and $\mathrm{WO}_{3}$ were milled for $2 \mathrm{~h}$, and then preheated at $950{ }^{\circ} \mathrm{C}$ for $3 \mathrm{~h}$ to form $\mathrm{Fe}_{2} \mathrm{WO}_{6}$. Afterward, $\mathrm{PbO}$ and $\mathrm{TiO}_{2}$ were mixed to the $\mathrm{Fe}_{2} \mathrm{WO}_{6}$, to form (1-x) $\mathrm{Pb}\left(\mathrm{Fe}_{2 / 3} \mathrm{~W}_{1 / 3}\right) \mathrm{O}_{3}-$ $\mathrm{xPbTiO}_{3}(\mathrm{PFW}-\mathrm{xPT})$. The mixture was initially calcined at $800^{\circ} \mathrm{C}$ for $3 \mathrm{~h}$, reground, pressed in pellets and finally sintered between $830-850{ }^{\circ} \mathrm{C}$ for $5 \mathrm{~h}$. The temperature ramps were controlled at $5{ }^{\circ} \mathrm{C} / \mathrm{min}$ for heating and $2{ }^{\circ} \mathrm{C} /$ min for cooling.

This procedure avoids the formation of pyrochlore phase (details can be found elsewhere ${ }^{11}$ ). The sintered ceramic bodies were cut in disc form and polished to a thickness of $0.5 \mathrm{~mm}$ and $2 \mathrm{~mm}$ in diameter. After that, they were annealed at $873 \mathrm{~K}$ for $0.5 \mathrm{~h}$ to release mechanical stresses introduced during the polishing. Gold electrodes were sputtered on the samples faces for dielectric measurements. Microwave dielectric measurements were carried out in the temperature range of 80-420 K (network analyzer HP-8719C), while low frequency measurements (HP 4194A) were performed in the range $15-500 \mathrm{~K}$. The temperature dependence of the and magnetic moment $\mathrm{m}$ ' $(\mathrm{T})$ were measured using a commercial SQUID magnetometer range of temperatures $4 \mathrm{~K}-600 \mathrm{~K}$.

\section{Results}

\subsection{Multiferroics properties}

In a previous work, we reported the structural and electrical proprieties of the system $\mathrm{Pb}\left(\mathrm{Fe}_{2 / 3} \mathrm{~W}_{1 / 3}\right) \mathrm{O}_{3}-\mathrm{PbTiO}_{3}$. The degree of $\mathrm{PbO}$ volatilization during the sintering of PFW was quite low, due to the low sintering temperatures. Relative densities of about 93-99 TD\% could be achieved in this route. XRD patterns of the PFW-PT ceramics indicate formation of a major phase with a cubic perovskite-type of structure (space group pm $\overline{3} \mathrm{~m}$ ). The average grain size was around $5 \mu \mathrm{m}$ for all samples and no systematic variation of grain size with change $\mathrm{x}$. All compositions with PT showed relatively high resistivity from $10^{7}$ to $10^{8} \Omega$.m. As can be seen the addition of PT increases the room temperature electrical resistivity of PFW samples. From these results, we concluded that all samples resulted in single phase, dense, with relatively high electrical resistivity, which allowed us to perform the dielectric and magnetic characterizations, which are presented and discussed in this work ${ }^{11}$.

Magnetic measurements, Figure 1a, show two characteristic temperatures $\left(T_{N 1}=340 \mathrm{~K}\right.$ and $\left.\mathrm{T}_{\mathrm{N} 2} \sim 15 \mathrm{~K}\right)$, which have been associated to two types of magnetic ordering for "pure" PFW. At $T_{N I}=340 \mathrm{~K}$ the a paramagnetic to antiferromagnetic ordering results from a strong superexchange $\mathrm{Fe}^{3+}-\mathrm{O}-\mathrm{Fe}^{3+}$ interactions. For the other one, a weak superexchange $\mathrm{Fe}^{3+}-\mathrm{O}-\mathrm{W}-\mathrm{O}-\mathrm{Fe}^{3+}$ interaction, caused by local short range order in the $\mathrm{B}$ site, is the responsible mechanism for the magnetic anomaly at lower temperatures (around $\mathrm{T}_{\mathrm{N} 2} \sim 15 \mathrm{~K}$ ). Similar results are shown in Figures 1b- 1d for the PFW-PT samples. As the amount of PT increases it can be observed that characteristic temperature $\mathrm{T}_{\mathrm{N} 1}$ decreases (from $340 \mathrm{~K}$ to $134 \mathrm{~K}$ for $x=0,0$ and $\mathrm{x}=0.30$, respectively). Otherwise the lower temperatures magnetic ordering occur at $T_{N 2}=15 \mathrm{~K}$ to $17 \mathrm{~K}$ for $x=0$ and $x=0.30$, respectively.

In Figure 2 the temperature of the imaginary relative dielectric permittivity ( $\varepsilon^{\prime}$ and imaginary $\varepsilon$ " components) for PFW-PT samples are shown as a function of the temperature at frequencies ranging from $1 \mathrm{KHz}$ to $1 \mathrm{MHz}$.

For PFW ceramic (Figure 2a) in the temperature range $150 \mathrm{~K}-400 \mathrm{~K}$ the dielectric permittivity shows at least two peaks in the real and imaginary components, which present a strong dependence on frequency. The peak around 180 $\mathrm{K}$, which can be attributed to a relaxor like ferroelectric ordering (FE), increases the temperature of maximum with the measuring frequency (Figure 2a). The dispersion
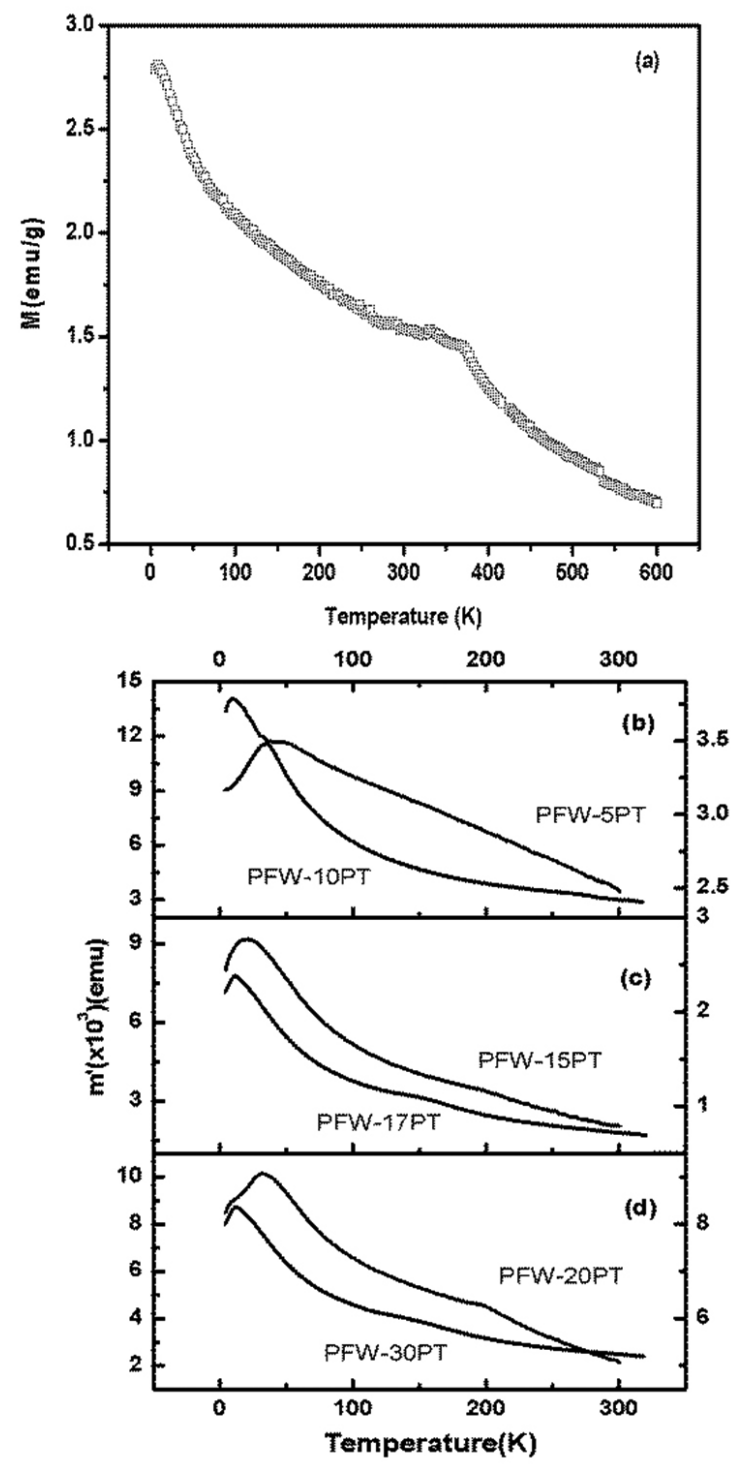

Figure 1. Temperature dependence of the magnetic moments $\mathrm{M}(\mathrm{T})$ (dc measurements) for PFW (a) and m'(T) (ac measurements) for PFW-PT system, $x=0.05$ and 0.10 (b), 0.15 and 0.17 (c) 0.20 and and $0.30(d)$. 
observed in the real and imaginary part in Figure 2, at temperatures higher than those related to the FE ordering, also shifts towards higher temperatures with increasing frequency, while the permittivity values decreased for higher frequencies characterizing a conductive contribution. As the conductive contributions do not alter the conclusions of this work it will not be no longer discussed. These results are also representative and similar to those measured in the other samples containing PT. Therefore, in Figure $2 \mathrm{~b}$ only the relative dielectric constant at $100 \mathrm{KHz}$ is shown for the PFW-PT samples. As the PT content in the (1-x)PFW$\mathrm{xPT}$ solid solutions is increased, the maximum dielectric permittivity, corresponding to the FE ordering increases up to $x=0.10$, decreasing for higher $\mathrm{x}$ values (Figure $2 \mathrm{~b}$ ). Also, the temperature corresponding to the maximum value of the dielectric constant increases from $\mathrm{T}=180 \mathrm{~K}$ for $\mathrm{x}=0$ (PFW) to $\mathrm{T}=320 \mathrm{~K}$ for $\mathrm{x}=0.3$ compound, while the character of the phase transition changes from diffuse, typical for relaxors, to a sharp one, characteristic of "normal" ferroelectrics.

\subsection{Microwave dielectric spectroscopy}

The frequency dependence of the real and the imaginary parts of the electrical permittivity for PFW ceramics is shown in Figure 3. Furthermore a well pronounced relaxation like dielectric dispersion is observed in the microwave region (around $1 \mathrm{GHz}$ ). As it can be seem the microwave dielectric dispersion does not vanish in the measured temperature

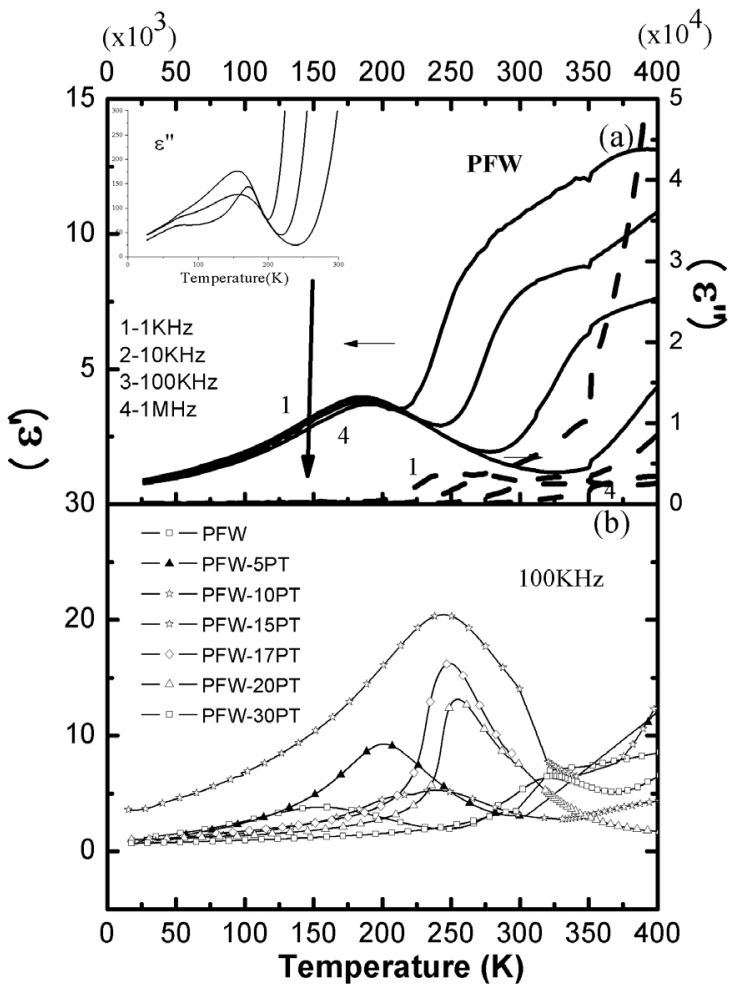

Figure 2. (a) Relative dielectric permittivity at various frequencies as a function of the temperature for PFW ceramic and at $100 \mathrm{kHz}$ (b) for $(1-x) \mathrm{Pb}\left(\mathrm{Fe}_{2 / 3} \mathrm{~W}_{1 / 3}\right) \mathrm{O} 3-x \mathrm{PbTiO}_{3}$ solid solutions $(x=0.1-0.30)$. interval, persisting even at temperatures as high as $200 \mathrm{~K}$ above $\mathrm{T}_{\mathrm{m}}$

The experimental data of Figure 3 can be fitted with a damped harmonic oscillator model as adopted for PLT ${ }^{12}$ ceramics, where $\varepsilon(f, T)$ takes the form:

$\varepsilon(f, T)=\varepsilon_{\mathrm{inf}}+\frac{\Delta \varepsilon f_{R}^{2}}{f_{R}^{2}-f^{2}+i \gamma f}$

where $\Delta \varepsilon(f, T)=\varepsilon\left(f_{0}, T\right)-\varepsilon\left(f_{\text {inf }} T\right)\left(\mathrm{f}_{0}<<\mathrm{f}_{\mathrm{R}}<<\mathrm{f}_{\text {inf }}\right)$ is the dielectric strength, $f_{R}(T)$ the characteristic frequency, $\gamma(T)$ a damping coefficient and $\varepsilon_{i n f}(T)$ the high-frequencies contribution by phonons and electronic polarization. $\varepsilon\left(f_{0}, T\right)$ should be interpreted here as the dielectric permittivity at frequencies lower than $f_{R}$, without any additional contribution (related to phase transitions or conductive mechanism).

The temperature dependence for $\Delta \varepsilon$ and $f_{R}$, reversible in the cooling and heating cycles, is shown in Figure 4. The observed temperature dependence of $\mathrm{f}_{\mathrm{R}}$ and $\Delta \varepsilon$ around $200 \mathrm{~K}, \mathrm{f}_{\mathrm{R}}$ reaches a minimum value, while $\Delta \varepsilon$ goes through a maximum, is associated with that of the dielectric permittivity peak, observed at RF range. Otherwise, remarkable are also the change of $f_{R}$ curve around $340 \mathrm{~K}$, and the set of "anomalies" around $250 \mathrm{~K}$ approximately, which are correlated to the secondary maximums of $\Delta \varepsilon$ at $340 \mathrm{~K}$ and around $250 \mathrm{~K}$ region, respectively. These

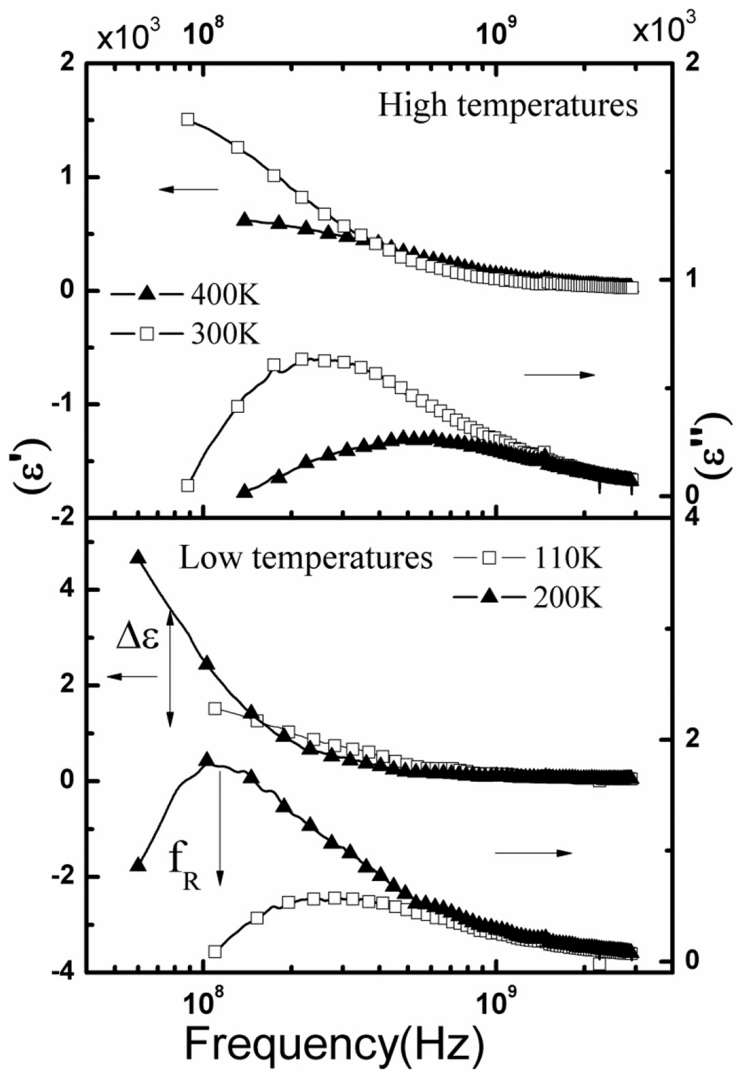

Figure 3. Temperature and frequency dependence of the real $(\varepsilon ')$ and imaginary $(\varepsilon ")$ components of the relative dielectric permittivity of PFW ceramics frequency dependence at various temperatures. 
secondary maximums cannot be detected in the radio frequency dielectric measurements. The dielectric anomaly observed around $340 \mathrm{~K}$ coincides with the temperature of the paramagnetic-antiferromagnetic ordering $\left(\mathrm{T}_{\mathrm{N} 1} \gg 340 \mathrm{~K}\right)$. Therefore, based on our previous results in multiferroic systems ${ }^{8,9,13,14}$, this dielectric anomaly in the PFW system results from a magnetostrictive contribution, due to a spinlattice coupling, in the paramagnetic-antiferromagnetic ordering.

Otherwise, the comparatively smaller anomaly detected around $250 \mathrm{~K}$, also observed in the magnetic measurements (Figure 2), must also be caused by magnetostrictive contribution (spin-lattice coupling) denominated as $\mathrm{T}_{\text {inst }}$ region. These instabilities were investigated through magnetic, anelastic and XANES measurements and the experimental data revealed that the observed magnetoelectroelastic instabilities, around $250 \mathrm{~K}$, and are associated with valence fluctuations of the iron ions in this region of temperature, configuring a charge-frustrated electronic structure ${ }^{15}$.

It can be seem (Figure 4b), at the magnetic ordering temperature, a decrease in $f_{R}$ is observed, followed by a decrease with the same previous trend. In fact, the dashed line that fits the points at temperatures below the antiferromagnetic ordering and above the ferroelectric, has the same slope of paramagnetic phase. Namely, the AFM ordering only caused a decrease in $f_{R}$, without modifying behaviorist temperature dependence. This would agree with the by Smolenskii's propose (despite not having
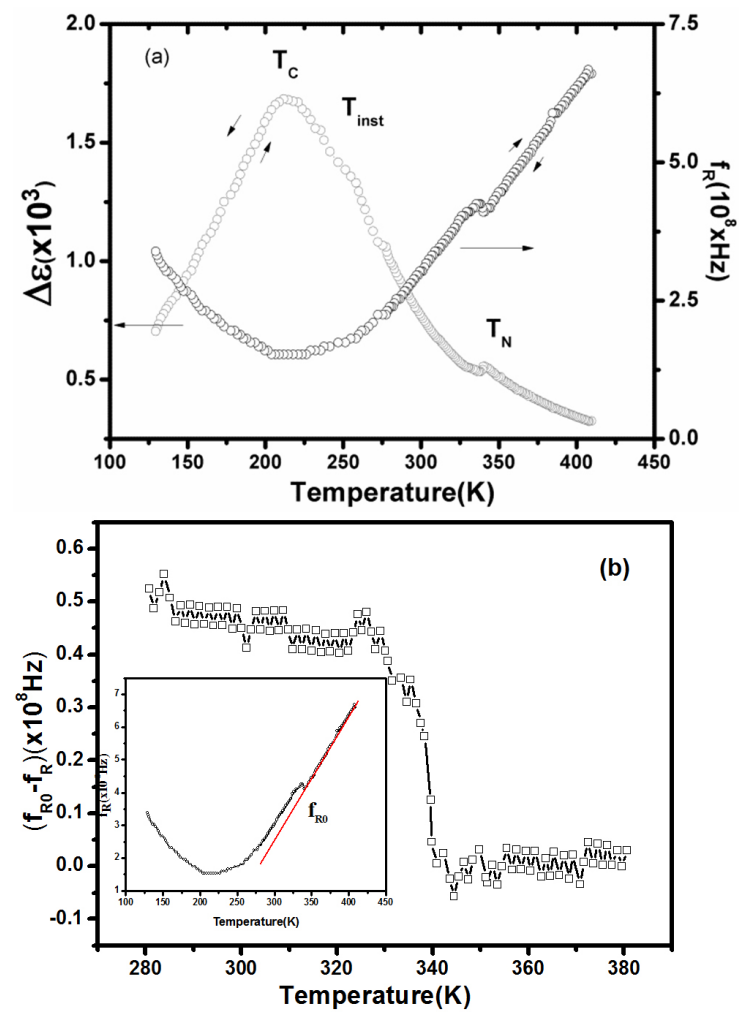

Figure 4. (a) Dielectric susceptibility at $80 \mathrm{MHz}$ and (b) dependence of the characteristic frequency ( $\mathrm{fR} 0$ - $\mathrm{fR}$ ) change versus temperature for PFW ceramics. experimental evidence): for materials where $T_{N}>T_{C}$, all the laws for ferroelectricity remain in effect, since the existence of AFM order will not influence the establishment of electric order ${ }^{16}$.

Generalizing these results for all PFW-PT samples, Figure 5 shows the temperature dependence for $\Delta \varepsilon$ and $f_{R}$ : all curves have a minimum in $\mathrm{f}_{\mathrm{R}}$ dependence and a maximum in $\Delta \varepsilon$ at the same temperature, correspond with FE phase transitions. In the same form, at $\mathrm{T}_{\mathrm{N}}$ all samples show a local maximum in the dielectric strength and a change in characteristic frequency.

Transition temperatures for ferroelectric (FE) and antiferromagnetic (AFM) phases are condensed in phase diagram of Figure 6. Néel and Curie temperatures of AFM and FE phases are indicated with different symbols for points measured with different techniques: magnetic ( $\square$ ) and dielectric $(\bullet)$ usual spectroscopy and by microwave dielectric technique $(\boldsymbol{\Delta})$.

First, the diagram shows how while the establishment of the temperature of magnetic ordering decreases, the ferroelectric transition temperature increases with the addition of $\mathrm{PbTiO}_{3}$ (PT) in the system. The temperatures where both orders coexist vary with the percentage of PT, and for some percentage between 10 and $15 \%$ to about $13 \%$ of PT, the two orders are simultaneously at temperature of approximately $235 \mathrm{~K}$. Although lower than room temperature, this is relatively high compared to most multiferroic materials in literature. Second, it can be seen

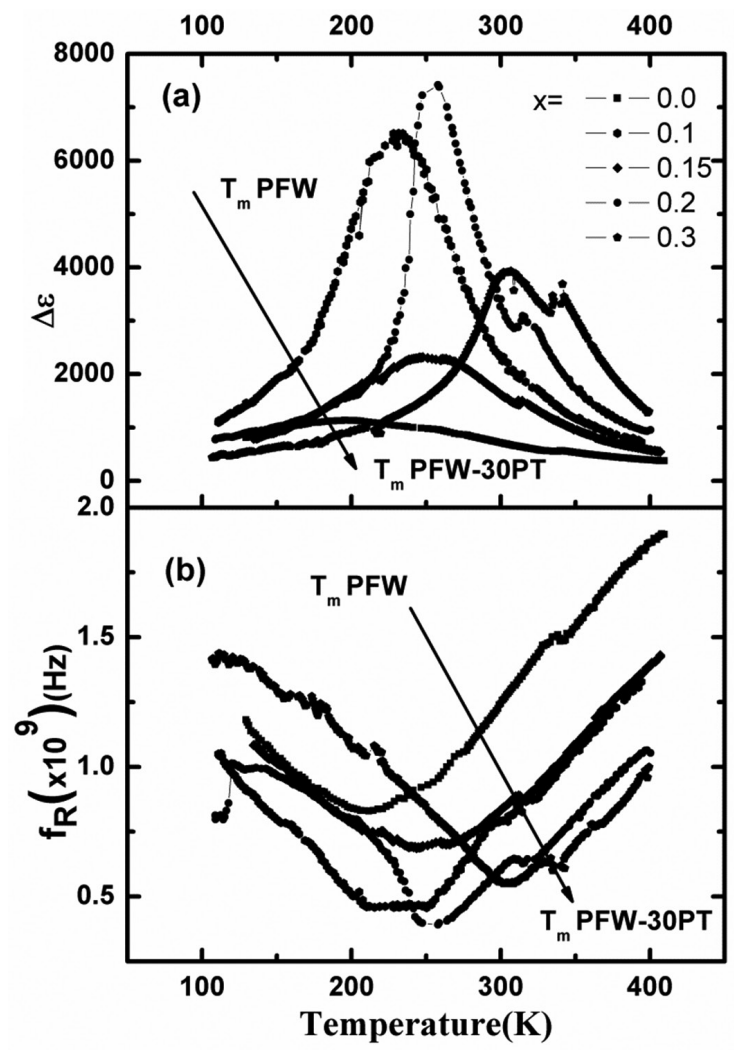

Figure 5. Temperature dependence of the characteristic coefficients of the microwave dispersion (a) characteristic frequency $\left(f_{R}\right)$ and dielectric strength $(\Delta \varepsilon)$ for PFW-PT ceramics. 


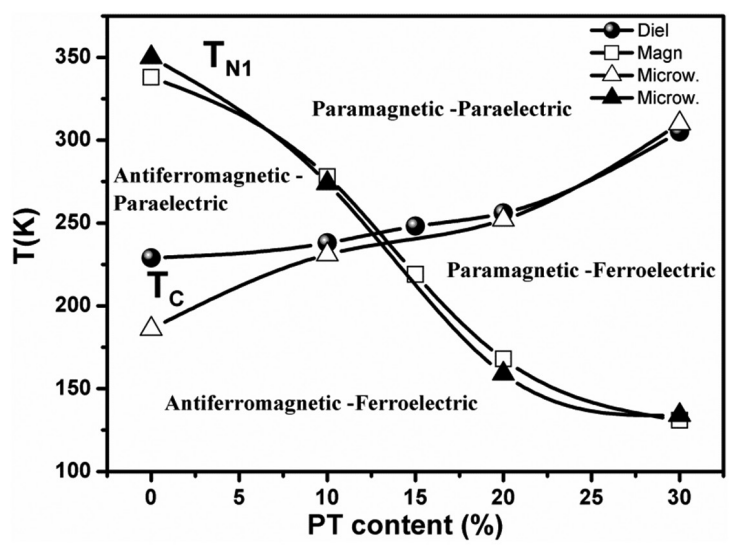

Figure 6. Transition temperatures for ferroelectric and antiferromagnetic ordering in $(1-\mathrm{x}) \mathrm{Pb}\left(\mathrm{Fe}_{2 / 3} \mathrm{~W}_{1 / 3}\right) \mathrm{O}_{3}-\mathrm{xPbTiO}_{3}$ samples, with $\mathrm{x}=0.0,0.05,0.10,0.15,0.17,0.20$ and 0.30 depending on the concentration of PT (x), measured by different techniques: dielectric impedance spectroscopy, magnetic susceptibility and dielectric microwave impedance spectroscopy.

that the temperatures measured by microwave dielectric techniques is very close for the observed by conventional measurements, confirm the effectiveness of this technique for detect both magnetic and ferroelectric phase transition.

Therefore, unless another unknown ordering superimposed over the magnetic ordering, this dielectric anomaly is caused by the magnetoelectric coupling in the PFW-PT system. This result demonstrates the high sensitivity of microwave dielectric measurements to probe weak transitions in bulk ceramics, as proposed before.

For last, we can observe a maximum in $\Delta \varepsilon$ at the same temperature of $\mathrm{T}_{\mathrm{N}}$ for the PFW samples, for all samples of the solid solution. What is surprising is that the maximum was

\section{References}

1. Eerenstein W, Mathur ND and Scott JF. Multiferroic and magnetoelectric materials. Nature. 2006; 442:759-765. PMid:16915279. http://dx.doi.org/10.1038/nature05023

2. Kreisel $\mathbf{J}$ and Kenzelmann M. MULTIFERROICS: The challenge of coupling magnetism and ferroelectricity. Europhysics News. 2009; 40:17-20. http://dx.doi.org/10.1051/ epn/2009702

3. Ramesh R. Materials Science: Emerging routes to multiferroics. Nature. 2009; 461: 1218-1219. PMid:19865157. http://dx.doi. org/10.1038/4611218a

4. Ivanov SA, Eriksson SG, Tellgrend R and Rundlofd H. Neutron powder diffraction study of the magnetoelectric relaxor $\mathrm{Pb}\left(\mathrm{Fe}_{2 / 3} \mathrm{~W}_{1 / 3}\right) \mathrm{O}_{3}$. Materials Research Bulletin. 2004; 39:23172328. http://dx.doi.org/10.1016/j.materresbull.2004.07.025

5. Guerra J de Los S, Lente MH and Eiras JA. Microwave dielectric dispersion process in perovskite ferroelectric systems. Applied Physics Letters. 2006; 88:102905-102905-3. http:// dx.doi.org/10.1063/1.2172072

6. Von Hippel A. Ferroelectricity, Domain Structure, and Phase Transitions of Barium Titanate. Reviews of Modern Physics. 1950; 22(3):221-37. http://dx.doi.org/10.1103/ RevModPhys.22.221 observed near $350 \mathrm{~K}$ not only in pure PFW but also in PFWxPT ceramics although $T_{N}$ should decrease with rising PT concentration. A decreased in $f_{R}$ was obtained for all samples at the same temperature. A similar behavior was obtained for acoustic emission for Dul'kin et al. ${ }^{15}$ and explained by different chemical compositions in the cores and shells of PFW-PT ceramic grains: Ti cations go practically only in the cores and the shells remain unchanged with Ti doping, i.e., two different magnetic phase transitions in the cores and the shells of PFW-xPT ceramics were expected, whereas the AFM temperature in the shells will be independent of PT concentration. In effect, the morphotropic phase boundary composition $0.68 \mathrm{PFW}-0.32 \mathrm{PT}$ were analyzed in situ by TEM as a function of temperature from 16 to $300 \mathrm{~K}$ for $\mathrm{Li}$ et al. ${ }^{16}$ and core-shell structure was detected at room temperature. Additional studies are necessaries to establish the origin of this phenomenon, but by this technique, we demonstrate the influence of the lattice dynamic at this temperature.

\section{Conclusions}

In conclusion, we investigated MW dielectric properties at microwave frequencies of $\mathrm{Pb}\left(\mathrm{Fe}_{2 / 3} \mathrm{~W}_{1 / 3}\right) \mathrm{O}_{3}-\mathrm{PbTiO}_{3}$ solid solutions, which confirmed the existence of elastic contribution at the temperatures of antiferromagnetic $\left(\mathrm{T}_{\mathrm{N}}\right)$ and ferroelectric $\left(\mathrm{T}_{\mathrm{C}}\right)$ ordering. The experimental results observed at MW frequencies measurements revealed clearly that the magnetic transition and instabilities can be correlated to spin-lattice coupling.

Acknowledgments

The authors wish to thank FAPESP for the financial support (Proc. 2008/04025-0 and 2010/11187-6) and to Mr. Francisco J. Picon and Mrs. Natalia A. Zanardi for the technical assistance.

7. Grigas J. Microwave Dielectric Spectroscopy of Ferroelectrics. Ferroelectrics. 2009; 380:113-121. http://dx.doi. org/10.1080/00150190902876249

8. Guerra JDS, Bassora LA and Eiras JA. Modelling the microwave dielectric response of 'normal' ferroelectrics. Solid State Communications. 2006; 140:426-429. http://dx.doi. org/10.1016/j.ssc.2006.09.014

9. Lente MH, Guerra JDS, De Souza GKS, Fraygola BM, Raigoza CFV, Garcia D et al. Nature of the magnetoelectric coupling in multiferroic $\mathrm{Pb}\left(\mathrm{Fe}_{1 / 2} \mathrm{Nb}_{1 / 2}\right) \mathrm{O}_{3}$ ceramics. Physical Review B. 2008; 78:054109-1- 054109-6.

10. Eiras JA, Fraygola BM and Garcia D. Structural and Dielectrics Properties of $(1-\mathrm{x}) \mathrm{Pb}\left(\mathrm{Fe}_{2 / 3} \mathrm{~W}_{1 / 3}\right) \mathrm{O}_{3-\mathrm{x}} \mathrm{PbTiO}_{3}$ Ceramics. Key Engineering Materials. 2010; 434-435:307-310. http://dx.doi. org/10.4028/www.scientific.net/KEM.434-435.307

11. Fraygola B, Coelho AA, Garcia D and Eiras JA. Magnetic and Dielectric Proprieties of Multiferroic (1-x) $\mathrm{Pb}\left(\mathrm{Fe}_{2 / 3} \mathrm{~W}_{1 / 3}\right)$ $\mathrm{O}_{3-\mathrm{x}} \mathrm{PbTiO}_{3}$ Ceramics Prepared Via a Modified Two-stage Solidstate Reaction. Materials Research. 2011; 14: 434-441. http:// dx.doi.org/10.1590/S1516-14392011005000075

12. Fraygola B, Mesquita A, Coelho AA, Garcia D, Mastelaro RV and Eiras JÁ. Evidence of Fe valence fluctuations and related magnetic and elastic instabilities in $\mathrm{Pb}$ based multiferroics perovskites. Journal of Applied Physics. 2012. 
13. Fraygola B, Garcia D and Eiras JA. Anelastic characterization in PFW multiferroics ceramics. Ferroelectrics. 2012. http:// dx.doi.org/10.1080/00150193.2012.675782

14. Smolenskii GA. Ferroelectromagnets. Uspekhi Fizicheskikh Nauk. 1982; 137:415-448. http://dx.doi.org/10.3367/ UFNr.0137.198207b.0415

15. Dul'kin E, Mojaev E, Roth M, Kamba S and Vilarinho PM. Burns, Neel, and structural phase transitions in multiferoic
$\mathrm{Pb}\left(\mathrm{Fe}_{2 / 3} \mathrm{~W}_{1 / 3}\right) \mathrm{O}_{3-\mathrm{x}} \mathrm{PbTiO}_{3}$ detected by an acoustic emission. Journal of Applied Physics. 2008;103:083542-083542-5. http:// dx.doi.org/10.1063/1.2907969

16. Li Z, Wu A, Vilarinho PM and Reaney IM. Core-shell microstructures in $0.68 \mathrm{~Pb}\left(\mathrm{Fe}_{2 / 3} \mathrm{~W}_{1 / 3}\right) \mathrm{O}_{3-0.32} \mathrm{PbTiO}_{3}$ at the morphotropic phase boundary. Journal of Physics-Condensed Matter. 2005; 17:2167-2175. http://dx.doi.org/10.1088/0953$8984 / 17 / 13 / 014$ 